\title{
Universal Infrared Absorption Spectroscopy Using Uniform Electromagnetic Enhancement
}

\author{
Sencer Ayas, ${ }^{\dagger}$ Gokhan Bakan, ${ }^{*},{ }^{\dagger}$ Erol Ozgur, $^{\dagger}$ Kemal Celebi, $^{\dagger}$ and Aykutlu Dana ${ }^{*}{ }^{\dagger}$ \\ ${ }^{\dagger}$ UNAM Institute of Materials Science and Nanotechnology, Bilkent University, 06800 Ankara, Turkey \\ ${ }^{\ddagger}$ Department of Electrical and Electronics Engineering, Antalya International University, 07190 Antalya, Turkey
}

\section{Supporting Information}

ABSTRACT: Infrared absorption spectroscopy has greatly benefited from the electromagnetic field enhancement offered by plasmonic surfaces. However, because of the localized nature of plasmonic fields, such field enhancements are limited to nanometer-scale volumes. Here, we demonstrate that a relatively small, but spatially uniform field enhancement can yield a superior infrared detection performance compared to the plasmonic field enhancement exhibited by optimized infrared nanoantennas. A specifically designed $\mathrm{CaF}_{2} / \mathrm{Al}$ thin film surface is shown to enable observation of stronger

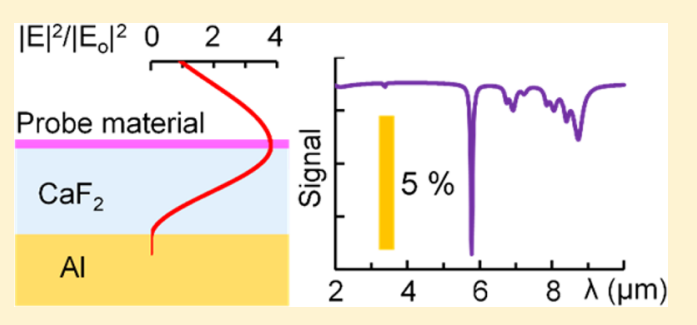
vibrational signals from the probe material, with wider bandwidth and a deeper spatial extent of the field enhancement as compared to such plasmonic surfaces. It is demonstrated that the surface structure presented here can enable chemically specific and label-free detection of organic monolayers using surface-enhanced infrared spectroscopy, indicating a great potential in highly sensitive yet cost-effective biomolecular sensing applications.

KEYWORDS: infrared absorption spectroscopy, protein sensing, vibrational spectroscopy, field enhancement, surface-enhanced infrared absorption

I nfrared absorption spectroscopy is a powerful method to directly probe the vibrational signatures of molecules, enabling label-free biochemical analysis. ${ }^{1,2}$ However, because of the poor interaction between the infrared field and nanoscale molecules, conventional infrared absorption methods require large numbers of molecules to collect significant information, making them insufficient to detect thin films or monolayers, which is crucial for biomolecular sensing. Some measurementbased modifications to infrared spectroscopy, such as attenuated total internal reflection ${ }^{3}$ or grazing angle techniques, ${ }^{4,5}$ enable monolayer detection, albeit still yielding low signal intensity and also requiring specialized optical setups or laborious sample preparation procedures. In order to overcome such problems, plasmonic field enhancement by surface nanostructuring has been widely studied in recent years, enabling the technique called surface-enhanced infrared absorption spectroscopy (SEIRA), which has been successfully demonstrated to enable monolayer detection. $^{6-11}$ However, like other plasmonic enhancement methods, SEIRA also requires patterned plasmonic structures that provide optical resonances close to the vibrational modes of the molecules, narrowing down the detection bandwidth. Hence, the resonance wavelength of the plasmonic structure has to be tuned, which can be done either physically by using lithography techniques $^{8}$ or, as recently demonstrated, electronically by employing patterned graphene electrodes. ${ }^{12}$ To overcome the need for tuning by increasing the bandwidth, spatially multiplexed designs with multispectral responses have also been demonstrated, $^{7}$ albeit requiring even more complex designs. Moreover, besides the narrow bandwidths and difficult fabrication, plasmonic field enhancement brings yet another fundamental problem: exponentially decaying electromagnetic fields, which reduce the active volume of detection. Therefore, even for the reports that claim signal enhancement factors of $10^{4}-10^{6}$, the far-field signal intensities are below $5 \%$. The reason for the discrepancy between the claimed enhancement factors and the signal intensity is the normalization values used for calculating the enhancement factors, which are merely the tiny nanoscale volumes at the antenna ends.

We anticipate that a simple thin film device that provides a small (near unity), but spatially uniform field enhancement can yield higher signal intensity values and much wider detection bandwidths compared to SEIRA, while not requiring any lithography. Here, we propose thin film structures for IR sensing and demonstrate the advantages of such structures versus conventional plasmonic structures (SEIRA), in terms of fabrication, signal intensity, bandwidth, and spatial detection range. We also present IR-based detection of self-assembled monolayers and proteins on these platforms.

A unity enhancement factor $\left(|E|^{2} /\left|E_{\mathrm{o}}\right|^{2}\right)$ can be achieved by suspending a nanometer-thick layer of a thin film (e.g., poly(methyl methacrylate) (PMMA)) in air, which enables the most basic infrared spectroscopy method. Here, the vibrational modes can be simulated as small dips in the transmission spectrum (Figure 1a). However, realizing a suspended layer of most materials over a spectroscopically measurable area (i.e., $\sim \mathrm{mm}^{2}$ ) is not easy. To overcome this difficulty, a thin $(\sim 50 \mathrm{~nm})$ suspended membrane, such as $\mathrm{SiN}_{x}$, can be used to act as a

Received: November 26, 2015

Published: February 4, 2016 


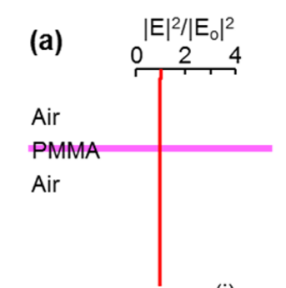

(i)

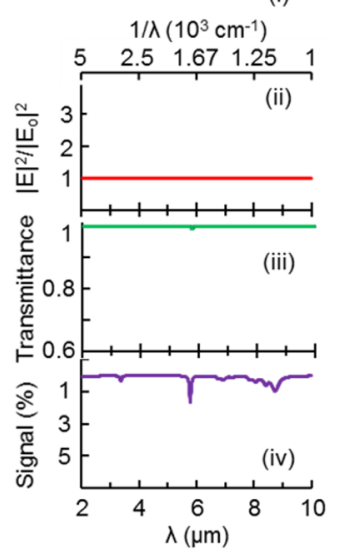

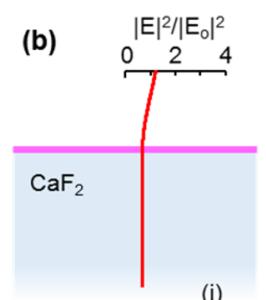

$1 / \lambda\left(10^{3} \mathrm{~cm}^{-1}\right)$

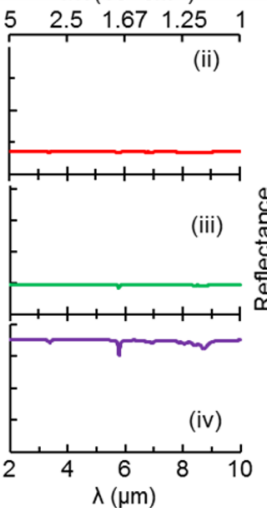

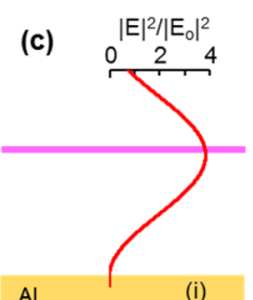

Al $1 / \lambda\left(10^{3} \mathrm{~cm}^{-1}\right)$
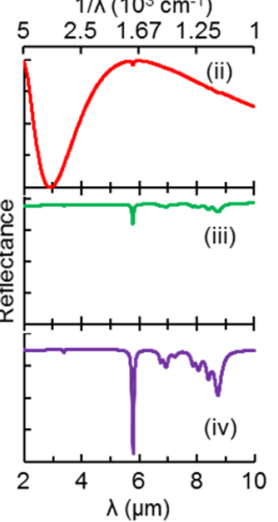

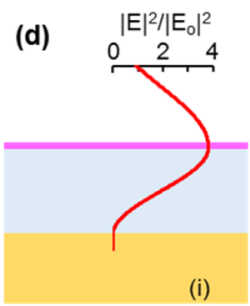

$1 / \lambda\left(10^{3} \mathrm{~cm}^{-1}\right)$
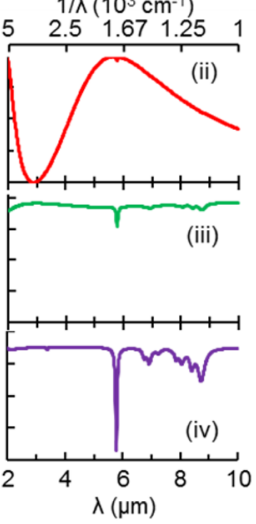

Figure 1. Calculated field profiles and IR transmittance/reflectance values for four basic thin film geometries. Columns: $10 \mathrm{~nm}$ PMMA is suspended in air (a), lies on a semicontinuous $\mathrm{CaF}_{2}$ substrate (b), is suspended $1.44 \mu \mathrm{m}$ above an $\mathrm{Al}$ surface (c), or lies on a $1 \mu \mathrm{m} \mathrm{CaF}{ }_{2}$-coated $\mathrm{Al}$ surface $(\mathrm{d})$. Rows: (i) electric field enhancement profiles as a function of the position through the cross sections, for $\lambda=5780 \mathrm{~nm}\left(1 / \lambda=1732 \mathrm{~cm}^{-1}\right)$; (ii) electric field enhancement factors at the bottom interfaces of the PMMA layers; (iii) far-field signal (transmittance/reflectance) spectra; (iv) absorption signals of the PMMA bands, which can be defined as the difference between the far-field signals with and without the PMMA layer. The optical properties of $\mathrm{CaF}_{2}$ have been measured for the simulations $(n=1.25$ in the IR; see Figure S6), and each PMMA band has been fitted to a Lorentzian oscillator (Figure S7).

support, while introducing other problems such as fragility and a strong signal background. The IR transmission measurements of a $10 \mathrm{~nm}$ PMMA-coated $50 \mathrm{~nm}$ thick $\mathrm{Si}_{3} \mathrm{~N}_{4}$ membrane show a detectable but small signal for the major PMMA band at $1732 \mathrm{~cm}^{-1}$ and very strong signals $(\sim 40 \%)$ for the $\mathrm{Si}-\mathrm{N}$ vibrational bands (Figure S1). To replace this fragile support, a thick IR transparent substrate (e.g., $\mathrm{CaF}_{2}$ or $\mathrm{MgF}_{2}$ ) can also be used. Such a layer reduces the enhancement factor from unity to 0.7 , but provides rigidity (Figure $1 \mathrm{~b}$ ). To obtain even higher enhancement factors, a reflective substrate, such as a metal layer, can be introduced. However, when a $10 \mathrm{~nm}$ PMMA layer is placed directly on the metal layer, no vibrational band signals can be obtained (Figure S2), unless the grazing angle method is used (Figure S3). On the metal, only if the PMMA layer becomes relatively thick $(>100 \mathrm{~nm})$ does the signal intensity reach $1.5 \%$ (Figure S4). So, when using a metal reflector, the maximum field intensity enhancement of 4 can be obtained at a distance of a quarter wavelength $(\sim \lambda / 4)$ away from the metal surface, ${ }^{13,14}$ because of the constructive interference of the incident and the reflected rays (Figure 1c). This configuration is similar to what is implemented in recent reports of electrically tunable graphene Salisbury screens in the $\mathrm{IR}^{13-15}$ and interferometric reflectance sensors in the visible. ${ }^{16,17}$ While such a configuration can be achieved by forming a thin membrane over a metal surface, the most rigid and trivial way is to use a dielectric coating. The enhancement factor at the air-dielectric interface in this case is given as

$$
\frac{|E|^{2}}{\left|E_{o}\right|^{2}}=(\underbrace{\left(\frac{r_{12}+r_{23} e^{2 i \beta}}{1+r_{12} r_{23} e^{2 i \beta}}\right)}_{r_{t}}+1)^{2}
$$

where $r_{x y}=\left(\tilde{n}_{x}-\tilde{n}_{y}\right) /\left(\tilde{n}_{x}+\tilde{n}_{y}\right), \tilde{n}$ is the complex refractive index, $\beta=(2 \pi / \lambda) n_{2} t$, $t$ is the thickness of the dielectric film, and layers 1,2 , and 3 are air, dielectric, and metal, respectively. For a dielectric layer with $\lambda / 4 n_{2}$ thickness, which makes the $e^{2 i \beta}$ term -1 , the total reflection coefficient $\left(r_{\mathrm{t}}\right)$ becomes 1 ; hence the enhancement factor becomes 4 if a perfect electrical conductor (PEC) is used as the reflector layer $\left(r_{23}=-1\right)$. However, although $\mathrm{Al}, \mathrm{Ag}$, and $\mathrm{Au}$ are the most reflective metals in the IR regime, they are not PECs (Figure S5). In such case, $r_{\mathrm{t}}$ is equal to $-r_{23}(\sim 1)$ for $n_{2}=1\left(r_{12}=0\right)$ (Figure 1c). When a dielectric with $n_{2}$ larger than 1 is used as the coating layer, $r_{\mathrm{t}}$ decreases owing to nonzero reflection from the top dielectric surface $\left(r_{12}<0\right)$ (Figure 1d). Hence, the enhancement factor is maximized $(\sim 4)$ when a low refractive index IR-transparent material such as $\mathrm{CaF}_{2}(n=1.2-1.4)$ with $\lambda_{\mathrm{V}} / 4 n_{2}$ thickness is used as the dielectric layer. Using an IR-transparent material with relatively high refractive index such as chalcogenides $(n \approx 4)$ reduces the enhancement to $\sim 3.6$. Therefore, Al can be the reflector of choice, because of its abundance and low cost, and $\mathrm{CaF}_{2}$ can be the dielectric layer, due to its IR transparency and low refractive index. The analysis of the $\mathrm{CaF}_{2} / \mathrm{Al}$ surfaces (Figure 1d) promises high signal intensity for an ultrathin probe material, a large bandwidth $(\sim 9 \mu \mathrm{m})$ that allows a variety of probe materials, and a large spatial extent of the field enhancement $(\sim 1 \mu \mathrm{m})$ that also enables the detection of thicker media, such as viruses.

An array of cross-shaped antennas on $\mathrm{Si}$ is designed as a reference plasmonic surface targeting the PMMA band at $1732 \mathrm{~cm}^{-1}$ following a SEIRA study on rod arrays. ${ }^{6}$ We first numerically compare the optical responses of the dielectric thin films on $\mathrm{Al}$ to those of the cross antenna arrays. Two main aspects of the surfaces are compared: the detection bandwidth and the spatial extent of the field enhancement. For the thin film surfaces, the 

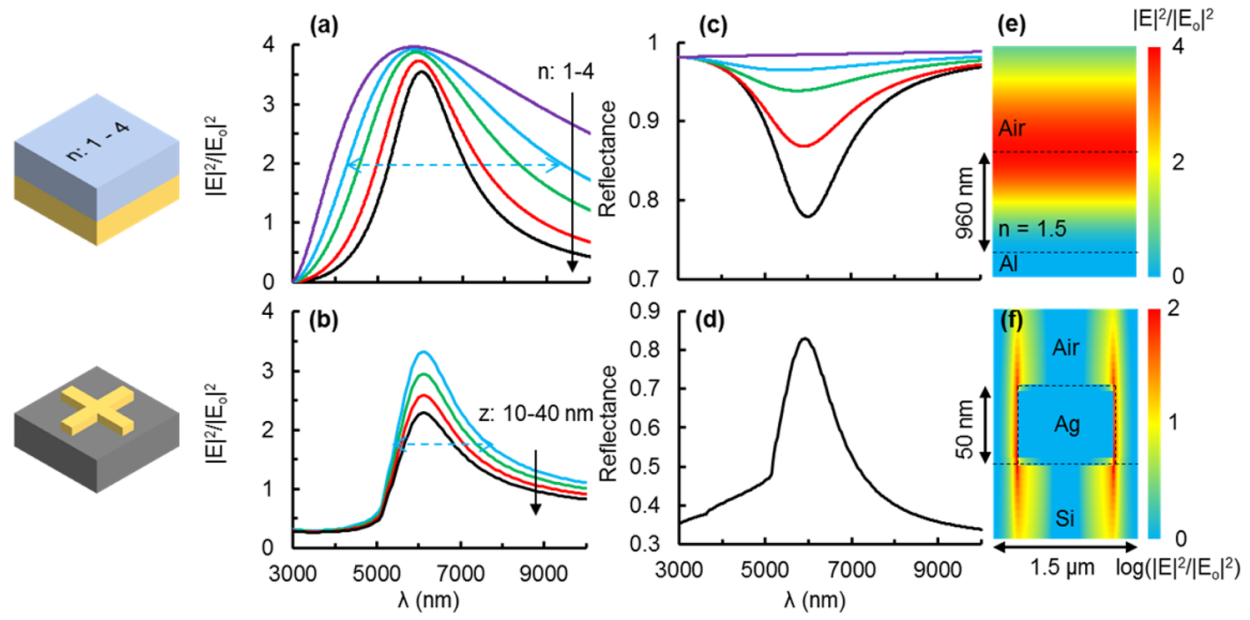

Figure 2. Numerical comparison of thin film surfaces to plasmonic antenna surfaces for IR spectroscopy. Field intensity spectra on the surfaces of various refractive index materials $\left(n=1,1.5,2,3,4\right.$; indicated by violet, light blue, green, red, and black curves, respectively) with $\lambda_{\mathrm{V}} / n$ thickness on $\mathrm{Al}$, where $\lambda_{\mathrm{V}}$ is $5780 \mathrm{~nm}$ (a). Averaged field intensity, from the antenna surface to the indicated maximum distance $(z)$ from the antenna (b). The dashed arrows mark the full-width at half-maximum bandwidths for $n=1.5 \mathrm{in}$ (a) and $z=10 \mathrm{~nm}$ in (b). The reflection spectra for the thin film surfaces (c) and the antennas (d). Field intensity distribution maps on the cross section of the dielectric-coated $(n=1.5, t=960 \mathrm{~nm}) \mathrm{Al}$ surface $(\mathrm{e})$ and the antenna (f), at the resonance wavelengths. Notice that (f) is not drawn to scale.

effect the dielectric layer's refractive index on these aspects is also investigated using hypothetical refractive indices ranging from 1 (air) to 4 (chalcogenides). The dielectric thickness is chosen as $\lambda_{\mathrm{V}} / 4 n\left(\lambda_{\mathrm{V}}=5.78 \mu \mathrm{m}\right)$, to address the major PMMA band. For increasing $n$, both the maximum enhancement factor at the dielectric layer's top surface and the bandwidth decrease (Figure 2a). For the case of $n=1.5$, which is close to the $\mathrm{CaF}_{2}$ refractive index, the full-width at half-maximum (fwhm) is $5.13 \mu \mathrm{m}$, while the fwhm for the antenna surface is much smaller $(2.44 \mu \mathrm{m})$. The field intensity spectrum for the antennas depends on the field-averaging volume. The field on and close to the surface is high; hence volume covering a small distance away from the surface yields larger average field intensities. For increasing volume, the average field intensity decreases owing to a quickly decaying field profile away from the surface (Figure 2b). The effect of increasing $n$ on the reflection spectra of the thin film surfaces is the appearance of deeper resonances owing to the increasing partial destructive interference of the light rays reflected from the dielectric-air and metal-dielectric interfaces (Figure $2 \mathrm{c}$ ). The same partial destructive interference is behind the reduction in the enhancement factor for increasing $n$. The resonance-free optical response of the dielectric film is more desirable for the infrared absorption spectroscopy, as the vibrational mode signal can be detected without any signal processing such as curve fitting and background subtraction. In contrast, such procedures are commonly required for plasmonics-based SEIRA, because the reflection/transmission spectra are nonuniform ${ }^{6}$ (Figure $2 \mathrm{~d}$ ). Moreover, the spatial extent of the field enhancement from the surface is very large for the dielectric surfaces, even close to $1 \mu \mathrm{m}$ (Figure 2e), enabling also the detection of thicker layers; meanwhile, the antennas' plasmonic field decays exponentially with a small decay length $(<100 \mathrm{~nm})$ (Figure $2 \mathrm{f})$, as also found previously. ${ }^{18}$

For the experimental comparison of the infrared absorption spectroscopy performances of the thin film surfaces and plasmonic structures, we have selected PMMA as the probe material, $\mathrm{Al}$ as the reflector, $\mathrm{CaF}_{2}$ as the dielectric, and $\mathrm{Ag}$ to form the plasmonic antennas that are defined by e-beam lithography (Figure S8). For the thin film measurements, the $\mathrm{CaF}_{2}$ thickness is chosen as $900 \mathrm{~nm}$, to have a field enhancement above unity at the IR range of $\lambda=3-12 \mu \mathrm{m}$. Thus, the reflection spectrum without PMMA is mainly flat above $\sim 3200 \mathrm{~cm}^{-1}$, as expected by the simulations (Figure S9), except the dips at 3400 and $1640 \mathrm{~cm}^{-1}$, which can be attributed to the $\mathrm{O}-\mathrm{H}$ modes (Figure 3a). This broad bandwidth enables easy identification of all the PMMA bands ranging from 3000 to $1000 \mathrm{~cm}^{-1}$, even when there is a very thin layer of PMMA on $\mathrm{CaF}_{2}$ (Figure 3a). The reflection from the $10 \mathrm{~nm}$ thick PMMA-coated antennas, however, shows only the major PMMA peak at $1732 \mathrm{~cm}^{-1}$ (Figure $3 \mathrm{~b}$ ) with lower signal intensity ( $3.5 \%$ vs $7.7 \%)$, as shown in the background-subtracted data in Figure $3 \mathrm{c}$. Besides the bandwidth and signal intensity, the spatial extent of the field enhancement by the $\mathrm{CaF}_{2}$ surfaces is much larger. Thus, when a thicker $(100 \mathrm{~nm})$ layer of PMMA is coated on both surfaces, the dip at $1732 \mathrm{~cm}^{-1}$ reaches $45 \%$ of the total reflection for the $\mathrm{CaF}_{2}$ surface, while the same dip is a mere $8 \%$ for the antenna surface (Figure 3d). The reflectance spectra used for Figure 3d are also provided in Figure S10.

Label-free detection performance for proteins and other organic molecules determine most of the application potential of an IR spectroscopy platform. We have used bovine serum albumin (BSA) and octadecanethiol (ODT) for the monolayer sensing tests (Figure 4). The BSA vibrational bands at 1652 (amide I) and $1531 \mathrm{~cm}^{-1}$ (amide II) are observed on the reflection spectrum with signal intensities of $6.5 \%$ and $2.7 \%$, respectively. Such signals are obtained by the difference in the IR reflection spectra of the $\mathrm{CaF}_{2} / \mathrm{Al}$ surfaces prepared using citrate solution with and without BSA (Figure S11). Here, the obtained signal intensity for amide I is much larger than the values reported on plasmonic surfaces for monolayer protein layers. ${ }^{6,10,19}$ Meanwhile, for ODT the vibrational bands are close to the edge of the enhancement bandwidth, at 2917 and $2849 \mathrm{~cm}^{-1}$, corresponding to asymmetric and symmetric $\mathrm{CH}_{2}$ stretching, respectively. Before the ODT adsorption, the $\mathrm{CaF}_{2}$ surface is functionalized by a $1.5 \mathrm{~nm}$ thick $\mathrm{Au}$ deposition, yielding Au nanoislands (Figure S12) instead of a continuous film, in order to minimize the effect of $\mathrm{Au}$ on the optical response (Figure S13). The $\mathrm{CaF}_{2} / \mathrm{Al}$ surface without $\mathrm{Au}$ nanoislands does not show significant ODT vibrational signal 

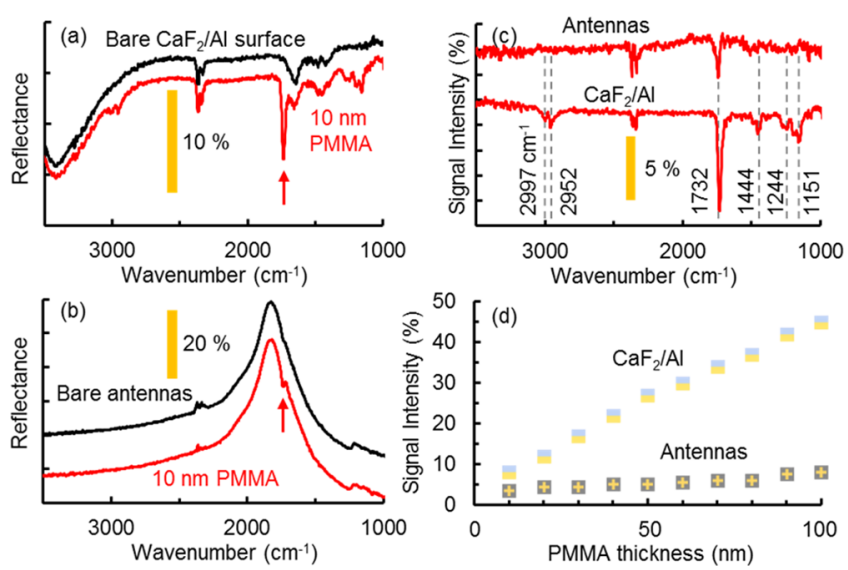

Figure 3. Experimental comparison of the $\mathrm{CaF}_{2} / \mathrm{Al}$ surface to the plasmonic antenna array, for IR spectroscopy. Reflection spectra of bare and $10 \mathrm{~nm}$ PMMA coated $\mathrm{CaF}_{2} / \mathrm{Al}$ surface (a) and plasmonic antenna surface (b). The signal intensities, defined as the difference between the PMMA-coated and bare surfaces, are shown in (c), displaying 7.7\% and 3.5\% PMMA signals at $1732 \mathrm{~cm}^{-1}$ for the $\mathrm{CaF}_{2} / \mathrm{Al}$ surface and the antennas, respectively. The thickness detection is compared in (d), where the PMMA dips at $1732 \mathrm{~cm}^{-1}$ are compared for both surfaces, while increasing the PMMA thickness from $10 \mathrm{~nm}$ to $100 \mathrm{~nm}$. The signal on the $\mathrm{CaF}_{2} / \mathrm{Al}$ surface shows a linear increase up to $45 \%$, which is much larger than the increase on the plasmonic surface $(8 \%$ vs $3.5 \%)$. Curves are shifted vertically for clarity in (a)-(c).

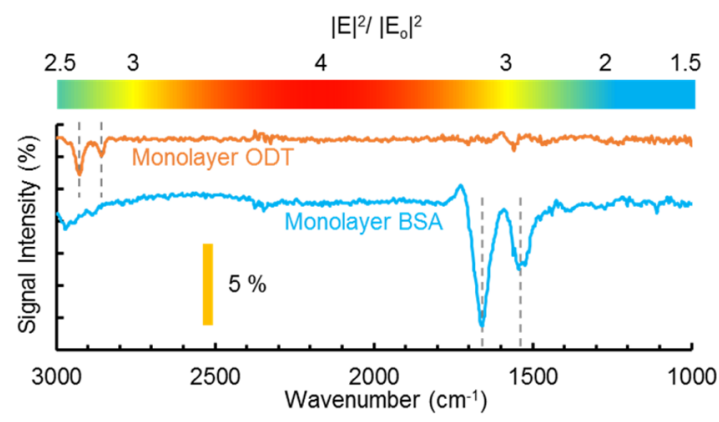

Figure 4. Monolayer sensing performance of the $\mathrm{CaF}_{2} / \mathrm{Al}$ surface. Measured signal intensities of monolayer BSA and ODT on the $\mathrm{CaF}_{2} / \mathrm{Al}$ surfaces after background subtraction (Figure S11). Calculated enhancement factor on the surface for $n=1.25$ and $t=900 \mathrm{~nm}$ is shown on top.

and, thus, acts as the background reference (Figure S11). On the Au-functionalized surface, the ODT bands at 2917 and $2849 \mathrm{~cm}^{-1}$ yield $2 \%$ and $1 \%$ reflection change, respectively (Figure 4). Despite a modest field enhancement factor of $\sim 2.5$ on the $\mathrm{CaF}_{2}$ surfaces at the active wavelengths, the measured signal intensities are comparable to the values from plasmonic surfaces optimized for the ODT vibrational bands. ${ }^{11,20,21} \mathrm{~A}$ state-of-the-art ATR system sandwiching a monolayer ODT layer between a Si substrate and Ge crystal can achieve a signal intensity of $3.3 \%$, being in the same range observed for the $\mathrm{CaF}_{2} / \mathrm{Al}$ and plasmonic surfaces. ${ }^{22}$

This study has investigated the use of interference-originated uniform field enhancement by flat thin films versus the plasmonic field enhancement by nanostructured metals, for the purposes of IR spectroscopy. The IR absorption signal intensities for the commonly used materials for IR probing (PMMA) on $\mathrm{CaF}_{2} / \mathrm{Al}$ thin film surfaces can exceed those on the plasmonic surfaces fabricated here and reported elsewhere. ${ }^{23,24}$ The detection bandwidths on the $\mathrm{CaF}_{2} / \mathrm{Al}$ platform have also been observed to be much larger than the plasmonic surfaces. Although there has been a recent report ${ }^{12}$ using graphene plasmonics to electrostatically tune the detection bandwidth for SEIRA between 1300 and $2000 \mathrm{~cm}^{-1}$, the simple $900 \mathrm{~nm}$ thick $\mathrm{CaF}_{2}$ film on $\mathrm{Al}$ can yield an above-unity enhancement factor in a larger range: $850-3300 \mathrm{~cm}^{-1}$. Such bandwidths can be further tuned by changing the dielectric thickness during deposition, which is much simpler than realizing the wavelength tuning on plasmonic surfaces, which requires controlling the shape and size of the lithographically defined features. The $\mathrm{CaF}_{2} / \mathrm{Al}$ surfaces, in contrast to the plasmonic surfaces, can also provide a large spatial extent of the field enhancement, which can be used to detect layers as thick as hundreds of nanometers. Moreover, the $\mathrm{CaF}_{2}$ surfaces do not exhibit Fano-type vibrational bands due to the strong coupling of plasmonic and vibrational modes, which necessitates complicated postprocessing techniques for the IR absorption data obtained on the plasmonic structures.

Besides the better IR detection performance, the proposed surfaces offer large-scale, low-cost, and easy fabrication, unlike the plasmonic surfaces, which typically require expensive lithography techniques on a limited area. The absence of patterning for the $\mathrm{CaF}_{2}$ surfaces also opens up new substrate possibilities such as $\mathrm{Al}$ foil and Al-coated plastics. A summary of the comparative study between the $\mathrm{CaF}_{2} / \mathrm{Al}$ surfaces and the fabricated antenna array is provided in Table 1 .

Table 1. Comparison of the $\mathrm{CaF}_{2} / \mathrm{Al}$ Surfaces and the Fabricated Antenna Array

\begin{tabular}{|c|c|c|}
\hline & $\mathrm{CaF}_{2} / \mathrm{Al}$ surfaces & plasmonic antennas \\
\hline lithography required? & no & yes \\
\hline \multirow[t]{3}{*}{ fabrication steps } & 1. Al deposition & 1. e-beam lithography \\
\hline & $\begin{array}{l}\text { 2. } \mathrm{CaF}_{2} \\
\text { deposition }\end{array}$ & 2. Ag deposition \\
\hline & & 3. lift-off \\
\hline size & wafer-scale & $100 \times 100 \mu \mathrm{m}^{2}$ \\
\hline tuning method & $\begin{array}{l}\text { varying } \mathrm{CaF}_{2} \\
\text { thickness }\end{array}$ & $\begin{array}{l}\text { varying the antenna sizes } \\
\text { (width, length, period) }\end{array}$ \\
\hline \multirow{2}{*}{$\begin{array}{l}\text { signal intensities for } 10 \text { and } \\
100 \mathrm{~nm} \text { PMMA layers }\end{array}$} & $7.7 \%$ & $3.5 \%$ \\
\hline & $45 \%$ & $8 \%$ \\
\hline $\begin{array}{l}\text { spatial extent of the field } \\
\text { enhancement }\end{array}$ & $900 \mathrm{~nm}$ & $<100 \mathrm{~nm}$ \\
\hline bandwidth & $3-12 \mu \mathrm{m}$ & $5-6 \mu \mathrm{m}$ \\
\hline
\end{tabular}

In conclusion, simple unpatterned dielectric/metal thin films, such as $\mathrm{CaF}_{2} / \mathrm{Al}$, can provide better IR detection performance, in terms of signal intensity, bandwidth, and spatial extent of the field enhancement, in comparison to the lithographically defined metal nanostructures that are typically used for SEIRA.

\section{METHODS}

Sample Preparation. $\mathrm{CaF}_{2}$ Surfaces. Al $(80 \mathrm{~nm})$ is deposited at a rate of $\sim 1 \AA / \mathrm{s}$ on Si wafers coated with a $3 \mathrm{~nm}$ Ge adhesion layer. Then, $\mathrm{CaF}_{2}$ films are deposited at a rate of $10 \AA / \mathrm{s}$. A thermal evaporation system is used for both evaporation steps. A $10^{-6}$ Torr pressure is reached before evaporation is started.

Ag Antenna Array. A $125 \mathrm{~nm}$ thick PMMA (MicroChem Nano 950k A2) is spin-coated on a $2 \mathrm{~cm} \times 2 \mathrm{~cm}$ silicon substrate. PMMA is prebaked at $180^{\circ} \mathrm{C}$ for $90 \mathrm{~s}$. Then e-beam lithography is performed with a FEI Nova NanoSEM electron microscope equipped with a Raith ElphyPlus system with an acceleration voltage of $30 \mathrm{kV}$ and beam current of $26 \mathrm{pA}$. 
PMMA is then developed using a MIBK/IPA (1:3) solution. PMMA patterning is followed by $1 \mathrm{~nm} \mathrm{Ge}$ (adhesion layer) and $50 \mathrm{~nm} \mathrm{Ag}$ deposition in a thermal evaporation system. The antenna array is obtained by a subsequent lift-off process in acetone. The area of the antenna array is $100 \mu \mathrm{m} \times 100 \mu \mathrm{m}$.

Ellipsometer Measurements. The thicknesses and optical properties of deposited $\mathrm{CaF}_{2}$ are characterized using variableangle visible spectroscopic ellipsometer measurements (J.A. Woollam Co., V-Vase). The Cauchy model is used to extract the optical properties in the measurement range and extrapolate toward the IR $(n=1.25)$. The thickness of PMMA layers spin-coated on silicon wafers is determined using variable-angle visible spectroscopic ellipsometer measurements. To obtain $\sim 10 \mathrm{~nm}$ PMMA films, PMMA in anisole solutions (PMMA $950 \mathrm{~K} \mathrm{~A} 2$ from MicroChem) is further diluted with toluene in a 1:4 ratio and then spin-coated at $8000 \mathrm{rpm}$. The optical parameters of PMMA layers are extracted using spectroscopic IR ellipsometer (J.A. Woollam Co., IR-Vase) measurements of a $130 \mathrm{~nm}$ PMMA film on a $\mathrm{Si}$ wafer using the Lorentzian oscillator model for each PMMA band.

Monolayer BSA Coating. The surfaces are incubated in a $1 \mathrm{mg} / \mathrm{mL}$ solution of BSA (Sigma-Aldrich) in citrate buffer solution ( $\mathrm{pH} \mathrm{4.0)}$ ) for $5 \mathrm{~min}$. Citrate buffer solution is freshly prepared from a mixture of aqueous citric acid $\left(\mathrm{C}_{6} \mathrm{H}_{8} \mathrm{O}_{7}\right.$, $0.1 \mathrm{M})$ and trisodium citrate $\left(\mathrm{Na}_{3} \mathrm{C}_{6} \mathrm{H}_{5} \mathrm{O}_{7}, 0.1 \mathrm{M}\right)$ solutions, 59:41 v/v. The samples are then rinsed first with citrate buffer and then distilled water and blow-dried with nitrogen.

Monolayer ODT Coating. The surfaces are immersed in a solution of $1 \mathrm{mM}$ ODT dissolved in ethanol absolute for $16 \mathrm{~h}$. The samples are then washed with ethanol to remove unattached molecules and blow-dried with nitrogen in order to form self-assembled monolayers on top of $\mathrm{Au}$ nanoislands.

FTIR Measurements. Bruker VERTEX 70 Fourier transform infrared spectroscopy with a Hyperion 2000 IR scanning microscope $(15 \times) \mathrm{NA}=0.4$ is used in either reflection or transmission mode. Background is collected from a bare aluminum surface for the reflection measurements. Transmittance of air is used as the reference for the transmission measurements. Measurements are performed with $2 \mathrm{~cm}^{-1}$ resolution and averaged over 64 scans for the $\mathrm{CaF}_{2} / \mathrm{Al}$ surfaces and 256 scans for the antennas. A knife edge aperture is used to collect light reflected from the patterned area for the antennas.

Simulations. Simulations are performed using the transfer matrix method for the $\mathrm{CaF}_{2} / \mathrm{Al}$ surfaces and a commercial FDTD package (from Lumerical) for the antennas. Threedimensional geometries with symmetric boundary conditions along the $x$-axis and asymmetric conditions along the $y$-axis are used. Perfectly matched layer boundary condition is used in the $z$-axis. A broadband plane wave $(\lambda=0.3-10 \mu \mathrm{m})$ is used to calculate the reflection spectrum and electric field profiles. The mesh size used in the simulations is $5 \times 5 \times 5 \mathrm{~nm}^{3}$. Dielectric functions used in the simulations are from the program's database (CRC data for $\mathrm{Al}$, Palik data for $\mathrm{Si}$ ) and our ellipsometer measurements (for $\mathrm{CaF}_{2}, \mathrm{PMMA}$ ). Native $\mathrm{SiO}_{2}$ and $\mathrm{Ge}$ adhesion layers are ignored in the simulations.

\section{ASSOCIATED CONTENT}

\section{S Supporting Information}

The Supporting Information is available free of charge on the ACS Publications website at DOI: 10.1021/acsphotonics.5b00680.

Figures S1-S4, infrared absorption spectroscopy of PMMA; Figure S5, IR reflection performance of metals;
Figures $\mathrm{S} 6$ and $\mathrm{S7}$, optical properties of $\mathrm{CaF}_{2}$ and PMMA; Figures S8 and S9, SEIRA using antennas; Figures S10 and S11, reflection spectra for PMMA and BSA on $\mathrm{CaF}_{2} / \mathrm{Al}$; Figures S12 and S13, SEM images and simulations of Au-nanoisland-covered $\mathrm{CaF}_{2} / \mathrm{Al}$ (PDF)

\section{AUTHOR INFORMATION}

\section{Corresponding Authors}

*E-mail (G. Bakan): gokhan.bakan@antalya.edu.tr.

*E-mail (A. Dana): aykutlu@unam.bilkent.edu.tr.

\section{Notes}

The authors declare no competing financial interest.

\section{ACKNOWLEDGMENTS}

This work is partially supported by TUBITAK grant no. 114E960 and EU FP7:People-IAPP NanoBacterPhageSERS.

\section{REFERENCES}

(1) Hartstein, A.; Kirtley, J. R.; Tsang, J. C. Enhancement of the Infrared Absorption from Molecular Monolayers with Thin Metal Overlayers. Phys. Rev. Lett. 1980, 45, 201-204.

(2) Kendall, C.; Isabelle, M.; Bazant-Hegemark, F.; Hutchings, J.; Orr, L.; Babrah, J.; Baker, R.; Stone, N. Vibrational Spectroscopy: A Clinical Tool for Cancer Diagnostics. Analyst 2009, 134, 1029-1045.

(3) Bassan, P.; Sachdeva, A.; Lee, J.; Gardner, P. Substrate Contributions in Micro-ATR of Thin Samples: Implications for Analysis of Cells, Tissue and Biological Fluids. Analyst 2013, 138, 4139-4146.

(4) Ishino, Y.; Ishida, H. Grazing Angle Metal-Overlayer Infrared ATR Spectroscopy. Appl. Spectrosc. 1988, 42, 1296-1302.

(5) Karabudak, E.; Kas, R.; Ogieglo, W.; Rafieian, D.; Schlautmann, S.; Lammertink, R. G. H.; Gardeniers, H. J. G. E.; Mul, G. Disposable Attenuated Total Reflection-Infrared Crystals from Silicon Wafer: A Versatile Approach to Surface Infrared Spectroscopy. Anal. Chem. 2013, 85, 33-38.

(6) Adato, R.; Yanik, A. A.; Amsden, J. J.; Kaplan, D. L.; Omenetto, F. G.; Hong, M. K.; Erramilli, S.; Altug, H. Ultra-Sensitive Vibrational Spectroscopy of Protein Monolayers with Plasmonic Nanoantenna Arrays. Proc. Natl. Acad. Sci. U. S. A. 2009, 106, 19227-19232.

(7) Aouani, H.; Rahmani, M.; Śípová, H.; Torres, V.; Hegnerová, K.; Beruete, M.; Homola, J.; Hong, M.; Navarro-Cía, M.; Maier, S. A. Plasmonic Nanoantennas for Multispectral Surface-Enhanced Spectroscopies. J. Phys. Chem. C 2013, 117, 18620-18626.

(8) Brown, L. V.; Zhao, K.; King, N.; Sobhani, H.; Nordlander, P.; Halas, N. J. Surface-Enhanced Infrared Absorption Using Individual Cross Antennas Tailored to Chemical Moieties. J. Am. Chem. Soc. 2013, 135, 3688-3695.

(9) Adato, R; Altug, H. In-Situ Ultra-Sensitive Infrared Absorption Spectroscopy of Biomolecule Interactions in Real Time with Plasmonic Nanoantennas. Nat. Commun. 2013, 4, 2154.

(10) Cetin, A. E.; Etezadi, D.; Altug, H. Accessible Nearfields by Nanoantennas on Nanopedestals for Ultrasensitive Vibrational Spectroscopy. Adv. Opt. Mater. 2014, 2, 866-872.

(11) Huck, C.; Toma, A.; Neubrech, F.; Chirumamilla, M.; Vogt, J.; De Angelis, F.; Pucci, A. Gold Nanoantennas on a Pedestal for Plasmonic Enhancement in the Infrared. ACS Photonics 2015, 2, 497505.

(12) Rodrigo, D.; Limaj, O.; Janner, D.; Etezadi, D.; Garcia de Abajo, F. J.; Pruneri, V.; Altug, H. Mid-Infrared Plasmonic Biosensing with Graphene. Science 2015, 349, 165-168.

(13) Jang, M. S.; Brar, V. W.; Sherrott, M. C.; Lopez, J. J.; Kim, L.; Kim, S.; Choi, M.; Atwater, H. A. Tunable Large Resonant Absorption in a Midinfrared Graphene Salisbury Screen. Phys. Rev. B: Condens. Matter Mater. Phys. 2014, 90, 165409.

(14) Thareja, V.; Kang, J.-H.; Yuan, H.; Milaninia, K. M.; Hwang, H. Y.; Cui, Y.; Kik, P. G.; Brongersma, M. L. Electrically Tunable 
Coherent Optical Absorption in Graphene with Ion Gel. Nano Lett. 2015, 15, 1570-1576.

(15) Brar, V. W.; Sherrott, M. C.; Jang, M. S.; Kim, S.; Kim, L.; Choi, M.; Sweatlock, L. A.; Atwater, H. A. Electronic Modulation of Infrared Radiation in Graphene Plasmonic Resonators. Nat. Commun. 2015, 6, 7032.

(16) Ozkumur, E.; Needham, J. W.; Bergstein, D. A.; Gonzalez, R.; Cabodi, M.; Gershoni, J. M.; Goldberg, B. B.; Unlü, M. S. Label-Free and Dynamic Detection of Biomolecular Interactions for HighThroughput Microarray Applications. Proc. Natl. Acad. Sci. U. S. A. 2008, 105, 7988-7992.

(17) Daaboul, G. G.; Yurt, A.; Zhang, X.; Hwang, G. M.; Goldberg, B. B.; Ünlü, M. S. High-Throughput Detection and Sizing of Individual Low-Index Nanoparticles and Viruses for Pathogen Identification. Nano Lett. 2010, 10, 4727-4731.

(18) Neubrech, F.; Beck, S.; Glaser, T.; Hentschel, M.; Giessen, H.; Pucci, A. Spatial Extent of Plasmonic Enhancement of Vibrational Signals in the Infrared. ACS Nano 2014, 8, 6250-6258.

(19) Chen, K.; Dao, T. D.; Ishii, S.; Aono, M.; Nagao, T. Infrared Aluminum Metamaterial Perfect Absorbers for Plasmon-Enhanced Infrared Spectroscopy. Adv. Funct. Mater. 2015, 25, 6637-6643.

(20) Cubukcu, E.; Zhang, S.; Park, Y.-S.; Bartal, G.; Zhang, X. Split Ring Resonator Sensors for Infrared Detection of Single Molecular Monolayers. Appl. Phys. Lett. 2009, 95, 043113.

(21) Cataldo, S.; Zhao, J.; Neubrech, F.; Frank, B.; Zhang, C.; Braun, P. V.; Giessen, H. Hole-Mask Colloidal Nanolithography for LargeArea Low-Cost Metamaterials and Antenna-Assisted Surface-Enhanced Infrared Absorption Substrates. ACS Nano 2012, 6, 979-985.

(22) Lummerstorfer, T.; Kattner, J.; Hoffmann, H. Monolayers at Solid-Solid Interfaces Probed with Infrared Spectroscopy. Anal. Bioanal. Chem. 2007, 388, 55-64.

(23) Wang, T.; Nguyen, V. H.; Buchenauer, A.; Schnakenberg, U.; Taubner, T. Surface Enhanced Infrared Spectroscopy with Gold Strip Gratings. Opt. Express 2013, 21, 9005.

(24) Abb, M.; Wang, Y.; Papasimakis, N.; de Groot, C. H.; Muskens, O. L. Surface-Enhanced Infrared Spectroscopy Using Metal Oxide Plasmonic Antenna Arrays. Nano Lett. 2014, 14, 346-352. 